\title{
Influence of hepatic ischemia-reperfusion on postoperative spatial cognitive function in mice
}

\author{
Y.Q. Wang, W.W. Wu, L.K. Wang, K. Chen and Y.H. Li \\ Department of Anesthesiology, \\ The First Affiliated Hospital of Anhui Medical University, Hefei, China \\ Corresponding author: Y.H. Li \\ E-mail: yuanhailicn@126.com \\ Genet. Mol. Res. 13 (3): 5767-5777 (2014) \\ Received June 5, 2013 \\ Accepted November 1, 2013 \\ Published July 29, 2014 \\ DOI http://dx.doi.org/10.4238/2014.July.29.4
}

\begin{abstract}
The aim of this study was to investigate the effects of partial hepatic ischemia/reperfusion (I/R) on postoperative cognitive function in mice. One hundred Kunming mice were randomized into control group $(\mathrm{N}=20)$, sham group $(\mathrm{N}=20)$ and $\mathrm{I} / \mathrm{R}$ group $(\mathrm{N}=60)$, which was equally divided into 3 subgroups according to the ischemia time (20,30 and $40 \mathrm{~min})$. Half of the mice in each group underwent a passive avoidance test on the 4th day, and the other underwent the test on the 18th day, which lasted for 6 days before euthanasia for analysis of brain pathology and immunohistochemistry for ChAT. The passive avoidance test showed that there was no significance in the incubation period and number of errors between the control and sham group, but there was a longer incubation period and more errors in the I/R group than control group; at G2, there was no significance between all groups. Hematoxylin-eosin staining of the hippocampus showed that at G1, there was no obvious change in hippocampal neurons in structure and arrangement except for IR/40 min; at G2, there was no significance between all groups. Immunohistochemistry of hippocampus for ChAT showed the following: at G1, there was no significance in average optical density of CA3 area between control and sham group, but optical density was significantly lower in I/R groups with I/R 40 min showing the lowest;
\end{abstract}


at G2, there was no significance between all groups. Pentobarbital has no effect on cognitive function, but hepatic partial ischemia and reperfusion injury does and could become worse over time.

Key words: Liver; Ischemia/reperfusion; ChaT; Postoperative cognitive dysfunction; Passive avoidance test

\section{INTRODUCTION}

Care for the postoperative patient is an important part of treatment. There are always two common methods of monitoring patients following surgery, clinical monitoring and general observation. Regular observations made assist to building a complete picture of the patient's condition following surgery and recovery from anesthesia. A structured assessment is usually carried out such as that described by Featherstone et al. (2008) where airway, breathing, circulation, disability (dysfunction of central nervous system) and exposure are assessed, and many areas use specific record sheets that allow all observations to be clearly recorded. Though postoperative care has been regarded seriously, the mental situation is missing in many care sheets.

Postoperative cognitive dysfunction (POCD) is one of the most important mental complications after surgery, which refers to the patients without preoperative mental disorders who are influenced by various postoperative factors leading to a postoperative reversible, fluctuant acute mental disorder syndrome including consciousness, cognition, memory, sense of orientation, psychomotor and sleep disorders. With the development of POCD, more and more patients have been diagnosed by neuropsychological examination, especially the memory test (Szwed et al., 2012).

Although POCD has become a common perioperative central nervous system complication, the mechanism is still unclear. The common causes for POCD were anesthesia and surgical trauma, especially cardiac surgery and neurosurgery, which usually lead to the ischemia/ reperfusion (I/R) injury. Hepatic surgery, which also can cause I/R injury, although causing distant organ injury such as to lung, intestine and kidney (Colletti and Green, 2006; Jildenstal et al., 2011; Kadkhodaee et al., 2012; Zhou et al., 2012), has not been studied much in that regard.

The study aimed at to investigate the influence of hepatic I/R injury on cognitive function. Since Geng et al. (2005) have reported that there is a similar short-term cognitive dysfunction caused by anesthesia-related trauma between mice and humans, the mouse was chosen as a POCD model. Besides the effects of $\mathrm{I} / \mathrm{R}$ injury on mouse cognitive function, the mechanism was also investigated by assaying choline acetyltransferase (ChAT), which is the rate-limiting enzyme of acetylcholine synthesis, since acetylcholine is a widespread neurotransmitter that is involved in recall and concentration (Neumeister and Riepe, 2012).

The mice were grouped by the different ischemic and recovery time to determine whether hepatic I/R injury can cause POCD in mice and if there is a time-dependency as well as ChAT expression.

\section{MATERIAL AND METHODS}

\section{Animals}

One hundred male Kunming mice (age: 2 months, weight: 18-22 g) in clean grade 
excluded by a step-through test were provided by Anhui Medical University, Experimental Animal Institute. This study was carried out in strict accordance with the recommendations in the Guide for the Care and Use of Laboratory Animals of the National Institutes of Health. The animal use protocol had been reviewed and approved by the Institutional Animal Care and Use Committee (IACUC) of the First Affiliated Hospital of Anhui Medical University. Before behavioral experiments, it took 2 days for mice to adapt to the laboratory environment, where they were allowed free access to water and food. The step-through test was conducted in a sound-proof environment at 8:00-12:00 every day at $25^{\circ} \mathrm{C}$.

\section{Animal grouping}

One hundred healthy adult male Kunming mice weighing 20-25 g excluded by the step-through test were randomly divided into 5 groups: control group $(\mathrm{N}=20)$, sham group $(\mathrm{N}=20$ ), group with ischemia for $20 \mathrm{~min}$ before reperfusion (IR/20 min, $\mathrm{N}=20$ ), group with ischemia for $30 \mathrm{~min}$ before reperfusion ( $\mathrm{IR} / 30 \mathrm{~min}, \mathrm{~N}=20$ ), group with ischemia for $40 \mathrm{~min}$ before reperfusion (IR/40 min, $\mathrm{N}=20$ ). Mice were fed normally without any treatment in the control group. The abdominal midline of mice in the sham group was incised and laparotomized, the hepatoduodenal ligament was separated, and the abdomen was then closed without any ischemic procedure. Ten of the mice sub-grouped from the group of IR/20 min, IR/30 min, and IR/40 min were subjected to the step-through test 10 days after surgery with the other 10 mice sub-grouped from the control and sham group, respectively. The rest of the 10 mice of each group underwent the step-through test starting on the 18th day for 6 days. Mice were sacrificed $1 \mathrm{~h}$ after the last tests for removal of brains. Tissue fixed in paraformaldehyde was used for hematoxylin-eosin (HE) staining and immunohistochemical analysis. The pathological changes of the hippocampus and ChAT enzyme expression in the hippocampus were observed.

\section{Establishment of the animal model}

Mice were allowed access to water, but not food, for $12 \mathrm{~h}$ preoperatively. After anesthesia with intraperitoneal injection of $3 \%$ amyl sodium pentobarbital, $30 \mathrm{mg} / \mathrm{kg}$, the middle of the upper abdominal was incised to expose hepatic portal. According to a literature method (Oliveira-Santos et al., 2012), atraumatic vascular clamps were used to block the left hepatic artery and portal vein to establish the partial the I/R injury model ( $70 \%$ liver parenchyma). The atraumatic vascular clamps were removed for reperfusion after ischemia for $20 \mathrm{~min}, 30$ min and $40 \mathrm{~min}$. The abdomen was then closed. Mice were kept under sterile conditions with intramuscular penicillin injection as anti-inflammatory treatment for 3 days after surgery. The tail arterial blood pressure (BP-98A and mouse non-invasive blood pressure meter) was monitored during the surgery. Rectal temperature was monitored continuously during ischemia. A heat lamp was utilized to maintain body temperature at $37-38.5^{\circ} \mathrm{C}$.

\section{Step-through test}

The experimental setup was divided into bright and dark rooms. There was a hole with a diameter of $3 \mathrm{~cm}$ between the two rooms. The copper gate at the bottom of the dark room was 
electrified. The mice were placed in the response box to adapt for $3 \mathrm{~min}$ (no electricity). In the test, the copper gate at the bottom of the dark room was connected to $40 \mathrm{~V}, 50 \mathrm{~Hz}$ AC. Mice were placed in the bright room with their backs against the hole. Once the mice entered the dark room, they were subjected to electric shocks. The first time when entering the dark room within 5 min (latent period, latent period longer than $180 \mathrm{~s}$ was excluded) and the frequencies of entrance (error frequency) were recorded. If the mouse had not entered the dark room within $5 \mathrm{~min}$, the latent period was defined as $300 \mathrm{~s}$ without error frequency.

\section{Statistical analysis}

The SPSS17.0 software was used for statistical analysis. The experimental data were expressed as means \pm standard deviation (means $\pm \mathrm{SD}$ ). The water maze test results were analyzed with repeated measures analysis of variance and multivariate analysis of variance of a general linear model. Paired comparisons of the different components at the same time were conducted. Other results were analyzed by single factor analysis of variance. $\mathrm{P}<0.05$ was considered to be statistically significant.

\section{RESULTS}

\section{Step-through test}

The results of step through test are shown in Tables 1 and 2. There was no statistical significance between the control group and sham group. The latent period and error frequencies of the IR/20 min and IR/30 min groups in the learning and training and memory test on the first, second and third day were greater than in the control group $(\mathrm{P}<0.05)$, while the IR/40 min group still showed greater values than the control group on the fourth and fifth day.

\section{HE staining of the hippocampus}

The one-week after surgery group (1-week group) was observed histopathologically under the light microscope. The mouse hippocampal neurons appeared to be arranged in an orderly manner with mild cytoplasmic edema and normal nucleus and chromatin in the IR/40 min group. Hippocampal neurons in other groups were arranged in an orderly manner with transparent cytoplasm and round or oval nucleus. Chromatin was distributed evenly with clear nucleoli and normal structure (Figure 1). There was no obvious abnormality in the 3rd week postoperatively in each group.

\section{ChAT enzyme immunohistochemical expression in the hippocampus}

After ChAT immunohistochemical staining of the hippocampus CA3 region 1 week postoperatively, optical density analysis showed that there was no statistical significance between the control group and sham group. Compared with the control group, statistical significance was found in the IR/20 min, IR/30 min, and IR/40 min groups. There was no statistical significance between the IR/20 $\mathrm{min}$ and IR/30 min groups. There was statistical significance between the IR/40 min group and the other 4 groups (Table 3). The number of ChAT-positive 


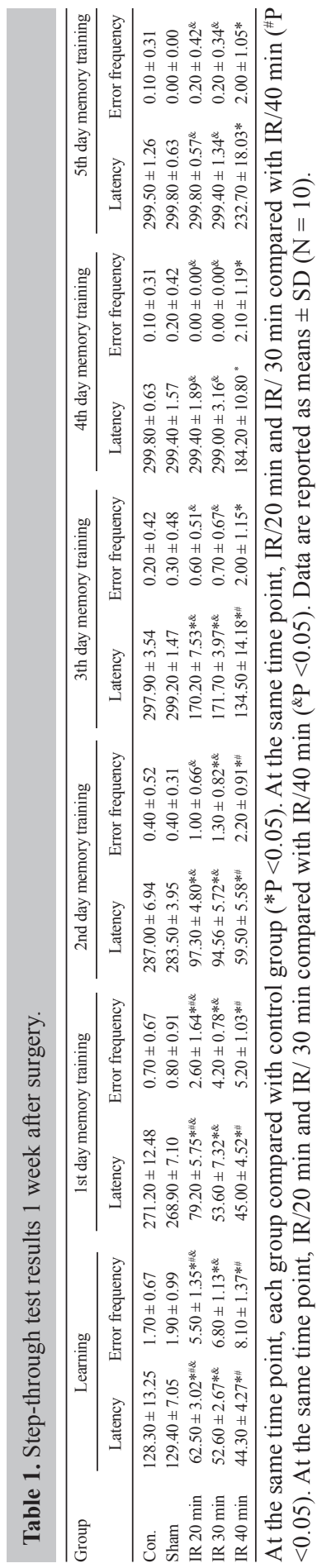


Y.Q. Wang et al.

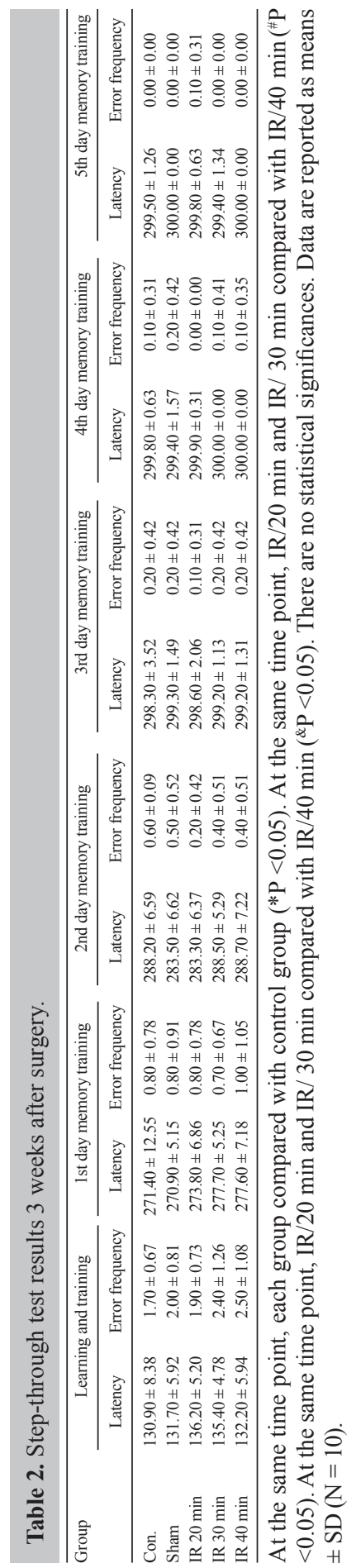


cells was lowest in the IR/40 min group. The result was consistent with the step-through test on the 3rd day (Figure 2). There was no statistical significance in ChAT-positive cell expression between groups in the 3rd week after surgery.

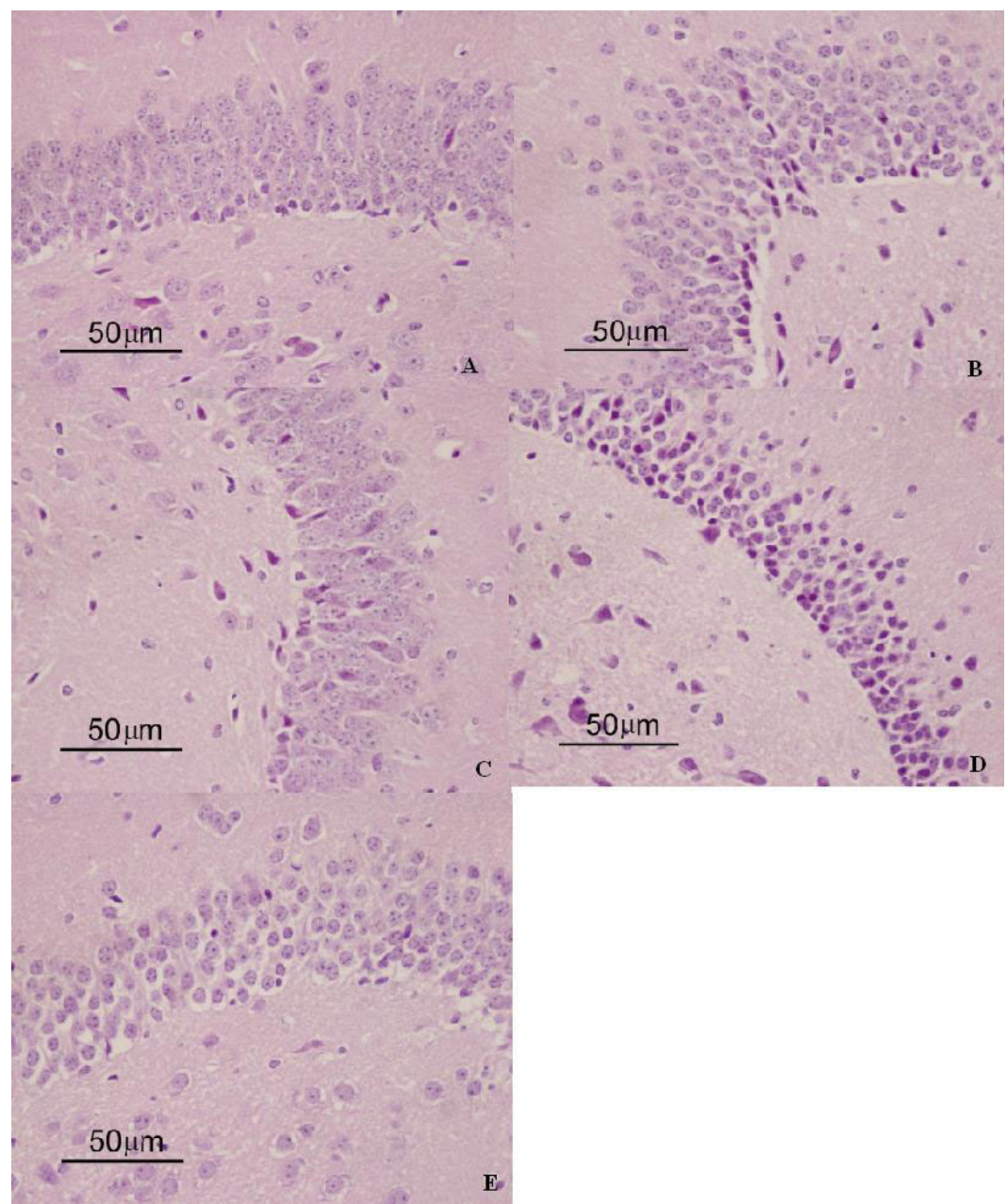

Figure 1. Hematoxylin and eosin staining of hippocampus in control group. A. Sham group; B. IR/20min; C. IR/30 $\mathrm{min}$; D. IR/40 min; E. groups 1 week postoperatively.

Table 3. Mean optical density of ChAT enzyme in hippocampus CA3 region one week after surgery.

\begin{tabular}{lcc}
\hline Group & Case (No.) & ChaT \\
\hline Con & 10 & $0.1932 \pm 0.0043^{\# \varepsilon}$ \\
Sham & 10 & $0.1907 \pm 0.0162^{* \varepsilon}$ \\
IR/20 min & 10 & $0.1523 \pm 0.0077^{* \&}$ \\
IR/30 min & 10 & $0.1550 \pm 0.0206^{* \&}$ \\
IR/40 min & 10 & $0.1433 \pm 0.0257^{* \#}$ \\
\hline
\end{tabular}

${ }^{*} \mathrm{P}<0.01=$ compared to control group; ${ }^{\#} \mathrm{P}<0.01=$ compare to $\mathrm{IR} / 30 \mathrm{~min}$ group; ${ }^{\&} \mathrm{P}<0.01=$ compared to IR/40 min group. Data are reported as means $\pm \mathrm{SD}$. 


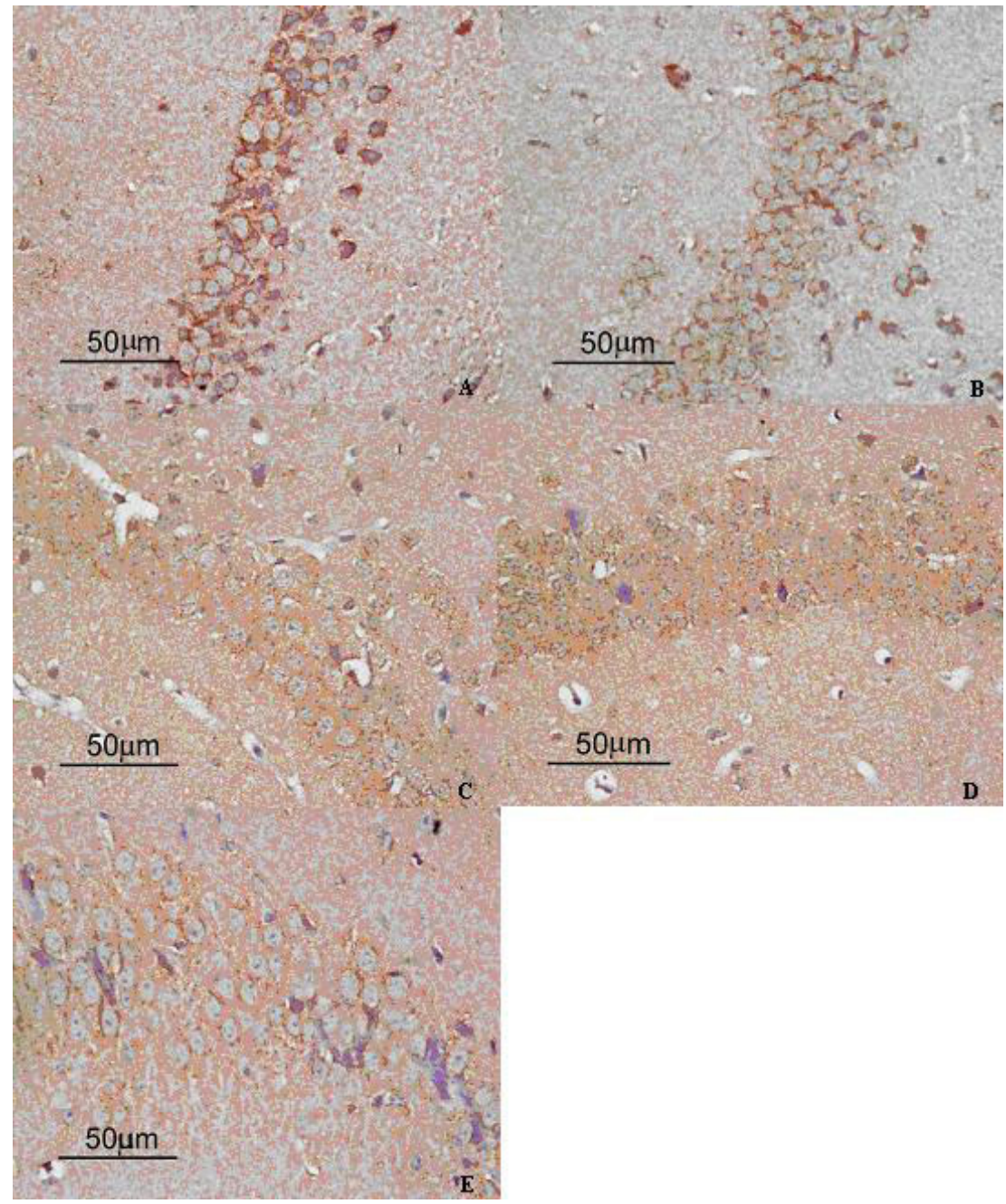

Figure 2. ChAT expression in the hippocampus (immunohistochemistry): in control group. A. Sham group; B. IR/20min; C. IR/30 min; D. IR/40 min; E. groups 1 week postoperatively.

\section{DISCUSSION}

Recently, POCD has become a hotspot of medical study. Its mechanism remains unclear. Various studies have confirmed that (Newman et al., 2007; Mason et al., 2010; Szwed et al., 2012) many factors are closely related to POCD, such as age, hypertension, diabetes, history of long-term medication, alcohol, education level, type of surgery, duration of anesthesia, intraoperative hypotension, intraoperative hypoxia, and postoperative pain. While the occurrence of POCD could be promoted by stress response, trauma, surgery, blood loss and blood transfusion, reduced cerebral blood flow, the formation of cerebrovascular microemboli, hypotension, postoperative hypoxemia, fluctuations in blood glucose and electrolyte imbalance. 
Nowadays, studies on POCD mechanism mainly focus on the anesthetic agents and surgical trauma. Grichnik et al. (1999) reported that $44.8 \%$ of patients still had cognitive dysfunction 6 to 12 weeks after surgery in their clinical study, and that even $24.7 \%$ showed severe cognitive impairment. Müller et al. (2004) believed that the type of surgery and invasion degree were closely related to cognitive dysfunction. In addition, other complications and mortality in the patient with POCD were also significantly increased.

In liver surgery, excessive bleeding, massive infusion and blood transfusion have a great effect on postoperative recovery (Gandhi and Sunder, 2012). Total hepatic vascular exclusion has been a common approach in liver resection to minimize blood loss, whereas the risk of hepatic ischemia/reperfusion injury (HIRI) has increased (Kelly et al., 2011). Hepatic I/R injury is a common pathological process in liver disease surgery, such as severe liver trauma, extensive liver resection and liver transplantation. There are a variety of reasons leading to hepatic I/R injury, which are mainly related to 3 factors: 1 ) the hypoxic injury during hepatic ischemia, 2) sinusoidal endothelial cells and liver cell damage after reperfusion, 3) the microcirculation secondary to endothelial cell injury. However, the explicit mechanism is not clear. Numerous studies have demonstrated that excessive release of inflammatory mediators, excessive generation of oxygen free radicals, calcium overload, programmed cell death and inflammatory factors possibly take part in this procedure. Hepatic $\mathrm{I} / \mathrm{R}$ is a systemic process that can cause organic and functional changes in lung, kidney, heart and other systems, especially the brain. Researchers (Gedik et al., 2012; Li et al., 2012; Wang et al., 2012) have found that lung inflammatory injury and acute myocardial damage caused by $\mathrm{I} / \mathrm{R}$ injury is related to the production of TNF- $\alpha$ after reperfusion. In a recent study, Zhou et al. (2012) found that the expression of TNF- $\alpha$ and IL-1 $\beta$ in lung and kidney tissue was significantly increased after hepatic I/R. It was believed that there was progressive impairment of cognitive function in patients 2 to 7 days after surgery, peaking on the 4th or 5th day (Kilo et al., 2001), and it was considered that a different degree of surgical invasion had a different influence on the cognitive function of surgical patients (Müller et al., 2004). However, there have been no reports of the influence of hepatic I/R on postoperative cognitive dysfunction.

The classic step-through test was adopted in this study to assess the cognitive function of 2-month-old mice. According to the study, there was no statistical significance in behavioral parameters between the control group and sham group. Short-term cognitive dysfunction appeared one week after surgery in hepatic I/R mice, especially on the 2nd, $3 \mathrm{rd}$, 4th, and 5th day. On the 3rd day, cognitive dysfunction and ischemia in each ischemia group appeared to be time-dependent, suggesting that the longer ischemia lasted, the worse the cognitive ability became. In the 3rd week after surgery, the behavioral experiments and hippocampus HE staining proved that cognitive abilities returned to normal in each mouse group.

Central cholinergic system plays an important role in the regulation of cognitive function such as learning, memory, and attention. ChAT, the rate-limiting enzyme for the synthesis of acetylcholine (a neurotransmitter that is directly related to cognition in the brain), is a marker enzyme of cholinergic nerves. In this experiment, ChAT-positive cells in the hippocampus CA3 region in each mouse group was consistent with the analysis of the memory test on the 3rd day in the behavioral tests, which was probably related to delayed expression of ChAT enzyme in the hippocampus. 
According to this study, anesthetics had no influence on cognitive function in mice postoperatively; meanwhile, hepatic I/R injury might have caused a short-term spatial cognitive dysfunction in mice, which appeared to be time-dependent in the step-through test. The longer the ischemia time, the worse the cognitive function was. When hepatic I/R occurs, plenty of hazardous substances produced by the liver including free radicals and inflammatory cytokines flow to the brain via the blood (Kimura et al., 2003). On the one hand, vascular endothelial cells in brain tissue are directly affected and brain cells suffer immediate damage (including mechanisms involving energy metabolism and blood-brain barrier). On the other hand, delayed damage is produced through the activation of the inflammatory response in brain tissue. The exact mechanism needs further study in the future.

\section{REFERENCES}

Colletti LM and Green M (2006). Lung and liver injury following hepatic ischemia/reperfusion in the rat is increased by exogenous lipopolysaccharide which also increases hepatic TNF production in vivo and in vitro. Shock 16: 312-319.

Featherstone P, Chalmers T and Smith GB (2008). RSVP: a system for communication of deterioration in hospital patients. Br. J. Nurs. 17: 860-864.

Gandhi R and Sunder R (2012). Postoperative analgesic efficacy of single high dose and low dose rectal acetaminophen in pediatric ophthalmic surgery. J. Anaesthesiol. Clin. Pharmacol. 28: 460-464.

Gedik HS, Korkmaz K, Erdem H, Karakilic E, et al. (2012). Protective effect of heparin in the end organ ischemia/ reperfusion injury of the lungs and heart. J. Cardiothorac. Surg. 7: 123.

Geng DQ, Wang HM, Wang ZP and Zeng YM (2005). Establishment and assessment of the rat model with postoperative cognitive dysfunction. J. Clin. Med. Pract. 9: 9-12.

Grichnik KP, Ijsselmuiden AJ, D’Amico TA, Harpole DH, Jr., et al. (1999). Cognitive decline after major noncardiac operations: a preliminary prospective study. Ann. Thorac. Surg. 68: 1786-1791.

Jildenstal PK, Hallen JL, Rawal N, Gupta A, et al. (2011). Effect of auditory evoked potential-guided anaesthesia on consumption of anaesthetics and early postoperative cognitive dysfunction: a randomised controlled trial. Eur. J. Anaesthesiol. 28: 213-219.

Kadkhodaee M, Mikaeili S, Zahmatkesh M, Golab F, et al. (2012). Alteration of renal functional, oxidative stress and inflammatory indices following hepatic ischemia-reperfusion. Gen. Physiol. Biophys. 31: 195-202.

Kelly DM, Shiba H, Nakagawa S, Irefin S, et al. (2011). Hepatic blood flow plays an important role in ischemia-reperfusion injury. Liver Transpl. 17: 1448-1456.

Kilo J, Czerny M, Gorlitzer M, Zimpfer D, et al. (2001). Cardiopulmonary bypass affects cognitive brain function after coronary artery bypass grafting. Ann. Thorac. Surg. 72: 1926-1932.

Kimura H, Katsuramaki T, Isobe M, Nagayama M, et al. (2003). Role of inducible nitric oxide synthase in pig liver transplantation. J. Surg. Res. 111: 28-37.

Li T, Zhang Z, Liao D, Chen Y, et al. (2012). The effect of polymerized placenta hemoglobin on renal ischemia/reperfusion injury. Artif. Cells Blood Substit. Immobil. Biotechnol. 40: 396-399.

Mason SE, Noel-Storr A and Ritchie CW (2010). The impact of general and regional anesthesia on the incidence of postoperative cognitive dysfunction and post-operative delirium: a systematic review with meta-analysis. J. Alzheimers. Dis. 22 Suppl. 3: 67-79.

Müller SV, Krause N, Schmidt M, Munte TF, et al. (2004). Cognitive dysfunction after abdominal surgery in elderly patients. Z Gerontol. Geriatr. 37: 475-485.

Neumeister KL and Riepe MW (2012). Synergistic effects of antidementia drugs on spatial learning and recall in the APP23 transgenic mouse model of Alzheimer's disease. J. Alzheimers. Dis. 30: 245-251.

Newman S, Stygall J, Hirani S, Shaefi S, et al. (2007). Postoperative cognitive dysfunction after noncardiac surgery: a systematic review. Anesthesiology 106: 572-590.

Oliveira-Santos M, Lopes MF, Catré D, Goncalves E, et al. (2012). Effect of hydroxyethyl starch on acute renal injury in a model of hepatic ischemia-reperfusion. Acta Med. Port. 25: 308-316.

Szwed K, Bielinski M, Drozdz W, Pawliszak W, et al. (2012). Cognitive dysfunction after cardiac surgery. Psychiatr. Pol. 46: 473-482.

Wang GZ, Yao JH, Jing HR, Zhang F, et al. (2012). Suppression of the p66shc adapter protein by protocatechuic acid prevents the development of lung injury induced by intestinal ischemia reperfusion in mice. J. Trauma Acute. Care 
Surg. 73: 1130-1137.

Zhou L, Yao X and Chen Y (2012). Dexamethasone pretreatment attenuates lung and kidney injury in cholestatic rats induced by hepatic ischemia/reperfusion. Inflammation 35: 289-296. 